\title{
Influence of Focal and Diffuse Extraprostatic Extension and Positive Surgical Margins on Biochemical Progression Following Radical Prostatectomy
}

\author{
Athanase Billis, Luciana L. Meirelles, Leandro L. L. Freitas, Luis A. Magna, Leonardo 0. Reis, Ubirajara
} Ferreira

Department of Pathology (AB, LLM, LLLF), Department of Medical Genetics/Biostatistics (LAM), and Department of Urology (LOR, UF), School of Medicine, University of Campinas (Unicamp), Brazil

\section{ABSTRACT}

Purpose: The amount of extraprostatic extension and positive surgical margin correlates in most studies with biochemical recurrence following radical prostatectomy. We studied the influence of focal and diffuse extraprostatic extension and positive surgical margins on biochemical progression using a simple method for quantification.

Materials and Methods: A total of 360 prostates were step-sectioned and totally processed from 175 patients with stage T1c and 185 patients with clinical stage T2 submitted to radical retropubic prostatectomy. Extraprostatic extension was stratified into 2 groups: present up to 1 quadrant and/or section from the bladder neck or apex (Group 1, focal) and in more than 1 quadrant or section (Group 2, diffuse); and, positive surgical margin present up to 2 quadrants and/or sections (Group 1, focal) and in more than 2 quadrants or sections (Group 2, diffuse). The Kaplan-Meier productlimit analysis was used for the time to biochemical recurrence, and an univariate and multivariate Cox stepwise logistic regression model to identify significant predictors. Results: Extraprostatic extension was found in 129/360 (35.8\%) patients, 39/129 (30.2\%) in Group 1 and 90/129 (69.8\%) in Group 2. In univariate analysis but not in multivariate analysis, patients showing diffuse extraprostatic extension (Group 2) had a significant higher risk to develop biochemical recurrence in a shorter time. Positive surgical margin was present in 160/360 (44.4\%) patients, 81/160 (50.6\%) patients in Group 1 and 79/160 (49.4\%) patients in Group 2. Patients with diffuse positive surgical margins (Group 2) had a significant higher risk in both univariate and multivariate analyses. Diffuse positive surgical margin was the strongest predictor on both analyses and an independent predictor on multivariate analysis.

Conclusion: Diffuse extraprostatic extension in univariate analysis and positive surgical margins on both univariate and multivariate analyses are significant predictors of shorter time to biochemical progression following radical prostatectomy.

\section{ARTICLE INFO}

\section{Key words:}

Prostatic neoplasms; extraprostatic extension; positive surgical margins; radical prostatectomy; treatment outcome

Int Braz J Urol. 2012; 38: 175-84

Submitted for publication: July 25,2011

Accepted after revision: October 29, 2011

\section{INTRODUCTION}

The amount of extraprostatic extension (EPE) and positive surgical margins (PSM) has prognostic importance in most studies (1-10).
However, during the International Society of Urological Pathology (ISUP) consensus conference on handling and staging of radical prostatectomy specimens held in Boston during the 98th meeting of the United States and Canadian Academy 
of Pathology (USCAP), no consensus could be reached as to how evaluate extent of EPE and PSM $(11,12)$. In this study we evaluated the influence of focal and diffuse extraprostatic extension and positive surgical margins on biochemical progression of patients submitted to radical prostatectomy using a simple method for EPE and PSM extent quantification that can be used in the daily practice of all pathologists who step-section and totally process the surgical specimen.

\section{MATERIALS AND METHODS}

This retrospective study was based on 360 consecutive patients with clinical stage T1c (175 patients) or T2 (185 patients) submitted to radical retropubic prostatectomy (RP) by one surgeon (UF). The clinicopathological variables studied included age at surgery, preoperative prostate-specific antigen (PSA) level, RP Gleason score, tumor extent at RP, EPE, PSM, seminal vesicle invasion and biochemical recurrence (BCR) following surgery.

After RP, serum PSA was drawn every 3 months during the first year, every 6 months dur- ing the second year, and annually thereafter. No patient of this series had radiotherapy or androgen manipulation before or after surgery. Only those with undetectable PSA after RP were included.

Total serum PSA was measured utilizing previous validated Immulite ${ }^{\circledR}$ PSA kit. BCR following surgery was considered as PSA $>0.2 \mathrm{ng} /$ $\mathrm{mL}$ according to recommendation of the American Urological Association (13). Patients without evidence of BCR were censored at last follow-up. The present study was approved by the Institutional Committee of Ethics of our Institution.

The surgical specimens were step-sectioned at 3 to $5 \mathrm{~mm}$ intervals and totally embedded in paraffin. A mean of 32 paraffin blocks were processed and $6 \mu \mathrm{m}$ sections from each block were stained with hematoxylin and eosin. Each transversal section of the prostate was subdivided into 2 anterolateral and 2 posterolateral quadrants (Figure-1A). Using the cone method, 8 sections from the bladder neck and 8 sections from the apex were obtained (Figure-1B). The number of sections obtained from the bladder neck and the apex were constant ( 8 from each

Figure 1. (A) Three transversal sections of the prostate specimen subdivided into 2 anterolateral and 2 posterolateral quadrants. (B) Eight sections from the bladder neck using the cone method.

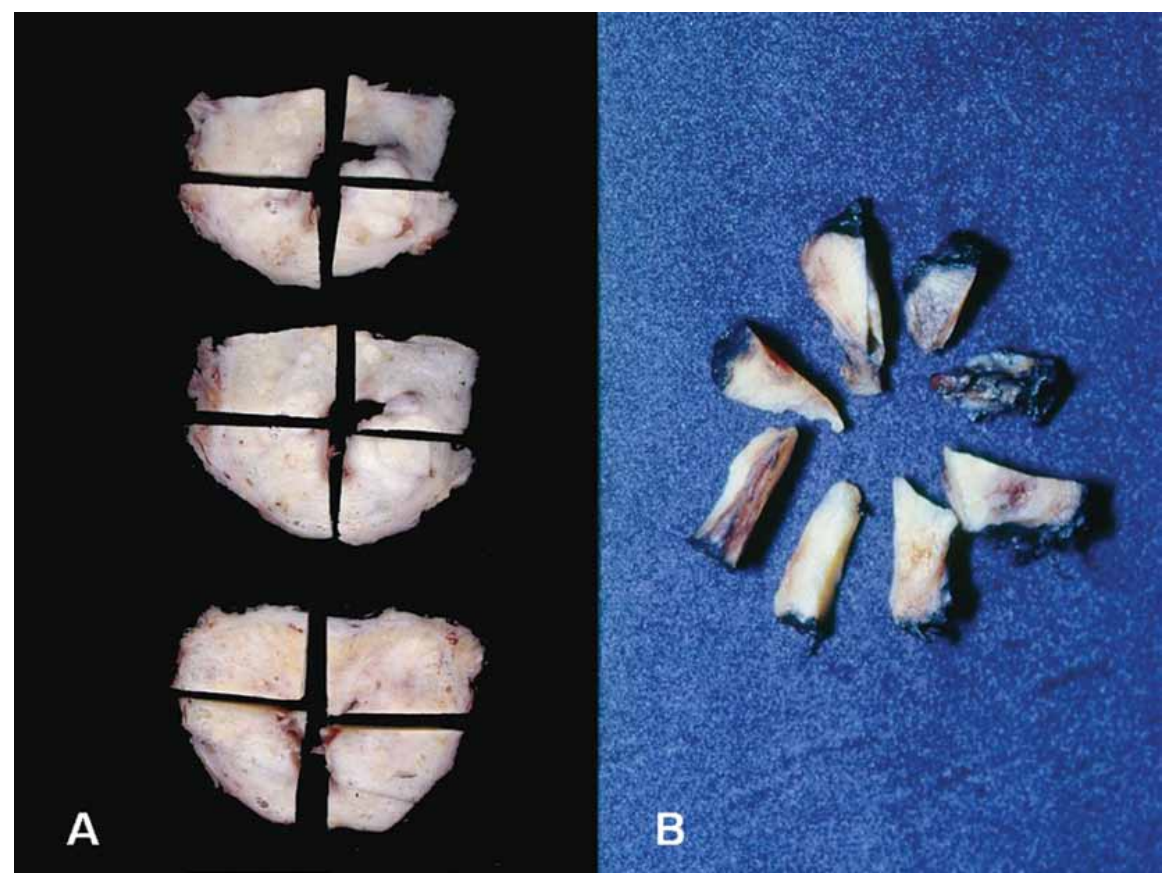


location), but the number of transversal sections varied according to the weight of the prostate. The median (range) of the prostate weight was $35 \mathrm{~g}(11 \mathrm{~g}-190 \mathrm{~g})$.

PSM was defined as cancer cells in contact with the inked specimen surface. EPE was diagnosed whenever cancer was observed in adipose tissue and, in case of desmoplastic response, whenever a protuberance corresponding to extension of tumor into periprostatic tissue was seen. Seminal vesicle invasion occurred whenever there was involvement of the muscular coat. Tumor extent at RP was evaluated by a semiquantitative point-count method previously described (14). Briefly, drawn on a sheet of paper, each quadrant of the transversal sections contained 8 equidistant points. During the microscopic examination of the slides, the tumor area was drawn on the correspondent quadrant observed on the paper. At the end of the examination the amount of positive points represented an estimate of the tumor extent. All cases were reviewed by a senior pathologist $(\mathrm{AB})$.

EPE was stratified into 2 groups: present up to 1 quadrant of the transversal sections and/ or sections from the bladder neck or apex (Group 1, focal EPE) and in more than 1 quadrant or sections (Group 2, diffuse EPE); PSM was stratified into 2 groups: present up to 2 quadrants and/or sections from the bladder neck or apex (Group 1, focal PSM) and in more than 2 quadrants or sections (Group 2, diffuse PSM).

\section{Statistical analysis}

The data were analyzed using the Fisher's exact test for comparison of proportions, the Mann-Whitney test for comparison of means, the Kaplan-Meier product-limit analysis for the time to biochemical recurrence (TBCR) using the logrank test for comparison between the groups, and an univariate and multivariate Cox stepwise logistic regression model to identify significant predictors of shorter TBCR. The P-values were two-sided at the significance level of $<0.05$. All statistical analyses were performed using the commercial available PASW Statistics (SPSS) 18.0.

\section{RESULTS}

\section{PATHOLOGICAL FINDINGS}

Extraprostatic extension. EPE was found in $129 / 360(35.8 \%)$ patients, 39/129 (30.2\%) in Group 1 and 90/129 (69.8\%) in Group 2. Comparing the clinicopathological features between the groups (Table-1) there was no significant difference related to age of the patients $(p=0.31)$. In Group 2, preoperative serum PSA $(p=0.02)$ and RP Gleason score $(p<0.01)$ were significantly higher, tumors were more extensive at RP ( $p=$ $0.04)$, and more tumors presented seminal vesicle invasion $(\mathrm{p}<0.01)$.

Positive surgical margin. PSM was present in 160/360 (44.4\%) patients. The single most frequently site involved was circumferential (quadrant of a transversal section). The second most frequently involvement was the concomitance of a quadrant and the apex. Only 3 specimens showed PSM concomitantly in the bladder neck, apex and circumferential. Considering extent of PSM, 81/160 (50.6\%) patients were in Group 1 and 79/160 (49.4\%) patients in Group 2. Comparing the clinicopathological features between the groups (Table-2), there was no significant difference related to age of the patients $(p=0.75)$, preoperative serum PSA ( $p=0.27)$, and seminal vesicle invasion $(\mathrm{p}=0.11)$. Group 2 tumors were more extensive at RP $(\mathrm{p}<0.01)$ and with higher RP Gleason score $(p=0.04)$.

\section{TIME TO BIOCHEMICAL RECURRENCE}

From the total of 360 men following radical prostatectomy, 115 (31.9\%) patients had BCR at a mean, median and range follow-up of 18, 9, and 3-129 months; 229 (62.1\%) censored men remained at risk at a mean, median and range followup of 46, 39, and 3-138 months, respectively; and, 16 (4.4\%) men had no serum PSA data.

Extraprostatic extension. At 5 years of follow-up, 69\% of patients without EPE vs 74\% of patients with EPE in Group 1 were free of BCR (logrank, $p=0.98$ ); and, $69 \%$ of patients without EPE vs 38\% of patients with EPE in Group 2 (log-rank, $\mathrm{p}<0.01$ ) (Figure-2). The weight of the prostates did not influence the results. Considering the median value of the weight, for prostates weighting 
Table 1 - Clinicopathological features comparing 39 patients with focal EPE (Group 1) vs. 90 patients with diffuse EPE (Group 2).

\begin{tabular}{|c|c|c|c|}
\hline Characteristic & $\begin{array}{l}\text { Focal EPE } \\
\text { (Group 1) }\end{array}$ & $\begin{array}{c}\text { Diffuse EPE } \\
\text { (Group 2) }\end{array}$ & $p$ Value \\
\hline \multicolumn{4}{|l|}{ Age (years) } \\
\hline mean $\pm \mathrm{SD}$ & $63.44 \pm 6.30$ & $63.30 \pm 6.38$ & $0.31^{*}$ \\
\hline median & 65 & 63.50 & \\
\hline range & $45-75$ & $47-74$ & \\
\hline \multicolumn{4}{|c|}{ Preoperative PSA } \\
\hline mean \pm SD & $9.43 \pm 4.72$ & $13.17 \pm 8.34$ & $0.02^{*}$ \\
\hline median & 8.2 & 12.0 & \\
\hline range & $2.3-21$ & $0.60-51$ & \\
\hline \multicolumn{4}{|c|}{ RP Gleason score } \\
\hline mean $\pm S D$ & $6.79 \pm 0.41$ & $7.39 \pm 0.74$ & $<0.01^{*}$ \\
\hline median & 7 & 7 & \\
\hline range & $6-7$ & $7-9$ & \\
\hline \multicolumn{4}{|c|}{ Tumor extension (positive points) } \\
\hline mean \pm SD & $50.94 \pm 37.94$ & $71.30 \pm 50.21$ & $0.04^{*}$ \\
\hline median & 36 & 50 & \\
\hline range & 7-192 & 9-225 & \\
\hline \multicolumn{4}{|c|}{ Seminal vesicle invasion } \\
\hline absent & $30(88.2 \%)$ & $37(58.7 \%)$ & $<0.01^{* *}$ \\
\hline present & $4(11.8 \%)$ & 26 (41.3\%) & \\
\hline
\end{tabular}

* Mann-Whitney test; ${ }^{* \star}$ Fisher's exact test

up to $35 \mathrm{~g}$, at 5 years of follow-up $67 \%$ of patients without EPE vs 83\% of patients with EPE in Group 1 were free of BCR (log-rank, $p=0.15$ ); and, 67\% of patients without EPE vs $43 \%$ of patients with EPE in Group 2 (log-rank, $p=0.03$ ). For prostates weighting $>35 \mathrm{~g}$, at 5 years of follow-up 71\% of patients without EPE vs 67\% of patients with EPE in Group 1 were free of BCR (log-rank, $p=0.12)$; and, $71 \%$ of patients without EPE vs $30 \%$ of patients with EPE in Group 2 (log-rank, $\mathrm{p}<0.01$ ).

Positive surgical margin. At 5 years of follow-up, 70\% of patients with negative margins vs $62 \%$ of patients with PSM in Group 1 were free of
BCR ( $=0.12)$; and, $70 \%$ of patients with negative margins vs 39\% of patients with PSM in Group 2 (log-rank, $p<0.01$ ) (Figure-3). This latter difference was also significant considering only specimens with pathologic stage T2 $(\mathrm{p}<0.01)$ as well as specimens pT2 and Gleason score $\leq 6(\mathrm{p}<0.01)$. No influence was found according to the location of PSM as well as weight of the prostates. For prostates weighting up to $35 \mathrm{~g}$, after 5 years of followup 69\% of patients without PSM vs 65\% of patients with PSM in Group 1 were free of BCR (log-rank, $\mathrm{p}=0.26$ ); and, $69 \%$ of patients without PSM vs $47 \%$ of patients with PSM in Group 2 (log-rank, $p$ 
Table 2 - Clinicopathological features comparing 81 patients with focal PSM (Group 1) vs. 79 patients with diffuse PSM (Group 2).

\begin{tabular}{|c|c|c|c|}
\hline Characteristic & $\begin{array}{c}\text { Focal PSM } \\
\text { (Group 1) }\end{array}$ & $\begin{array}{c}\text { Diffuse PSM } \\
\text { (Group 2) }\end{array}$ & $\mathrm{p}$ Value \\
\hline \multicolumn{4}{|l|}{ Age (years) } \\
\hline mean $\pm S D$ & $63.03 \pm 6.63$ & $63.53 \pm 7.15$ & $0.75^{\star}$ \\
\hline median & 64 & 64 & \\
\hline range & $47-74$ & $43-75$ & \\
\hline \multicolumn{4}{|c|}{ Preoperative PSA } \\
\hline mean $\pm S D$ & $9.91 \pm 6.49$ & $11.00 \pm 7.07$ & $0.27^{\star}$ \\
\hline median & 8.74 & 7.07 & \\
\hline range & $0.90-35$ & $1.22-51$ & \\
\hline \multicolumn{4}{|c|}{ RP Gleason score } \\
\hline mean $\pm S D$ & $6.81 \pm 0.56$ & $7.05 \pm 0.76$ & $0.04^{*}$ \\
\hline median & 7 & 7 & \\
\hline range & $5-9$ & $5-9$ & \\
\hline \multicolumn{4}{|c|}{ Tumor extension (positive points) } \\
\hline mean \pm SD & $38.86 \pm 31.72$ & $60.61 \pm 47.26$ & $0.01^{*}$ \\
\hline median & 27.50 & 45 & \\
\hline range & $1-147$ & $4-225$ & \\
\hline \multicolumn{4}{|c|}{ Seminal vesicle invasion } \\
\hline absent & $52(64.2)$ & $40(50.6)$ & $0.11^{* *}$ \\
\hline present & $29(35.8)$ & 39 (49.4) & \\
\hline
\end{tabular}

${ }^{*}$ Mann-Whitney test; ${ }^{* *}$ Fisher's exact test

$<0.01$ ). For prostates weighting $>35 \mathrm{~g}$, at 5 years of follow-up 78\% of patients without PSM vs 59\% of patients with PSM in Group 1 were freee of BCR (log-rank, $p=0.21$ ); and, 78\% of patients without PSM vs $28 \%$ of patients with PSM in Group 2 (logrank, $\mathrm{p}<0.01$ ).

\section{RISK FOR SHORTER TIME TO BIOCHEMI-}

\section{CAL RECURRENCE}

In univariate Cox regression analysis, age ( $p$ $=0.64)$, EPE Group $1(p=0.98)$, and PSM Group 1 $(p=0.12)$ were not significantly predictive of shorter TBCR. EPE Group 2 ( $p<0.01$ ), PSM Group 2 ( $p<$
0.01), preoperative PSA ( $p<0.01)$, RP Gleason score ( $p<0.01)$, RP tumor extent $(p<0.01)$, and seminal vesicle invasion $(\mathrm{p}<0.01)$ were significantly predictive of shorter TBCR (Table-3). PSM Group 2 was the strongest predictor. In multivariate analysis, the best model predictive of shorter TBCR was PSM Group $2(\mathrm{p}<0.01)+$ preoperative PSA $(\mathrm{p}<0.01)+$ seminal vesicle invasion $(p=0.04)$.

\section{DISCUSSION}

Several studies have shown that the amount of EPE has prognostic importance. Our 
Figure 2 - Kaplan-Meier biochemical (PSA) progression-free survival curve comparing 219 surgical specimens without EPE vs 37 surgical specimens with focal EPE (Group 1), and 88 surgical specimens with diffuse EPE (Group 2).

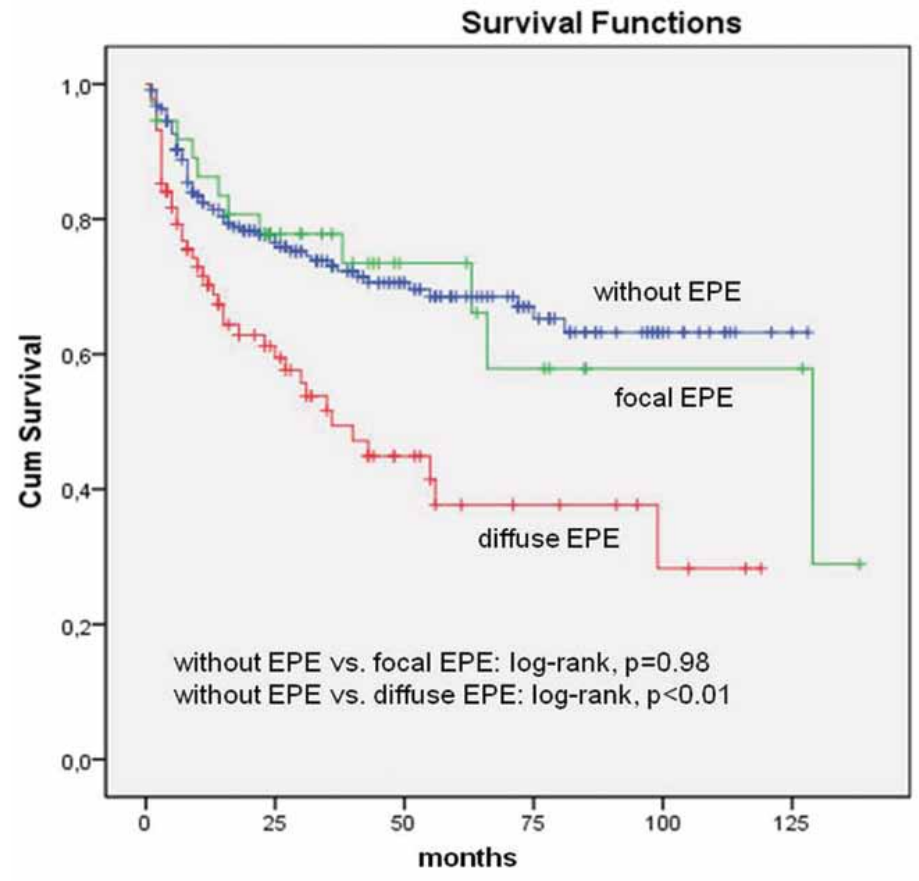

Figure 3 - Kaplan-Meier biochemical (PSA) progression-free survival curve comparing 183 surgical specimens without PSM vs 81 surgical specimens with focal PSM (Group 1), and 78 surgical specimens with diffuse PSM (Group 2).

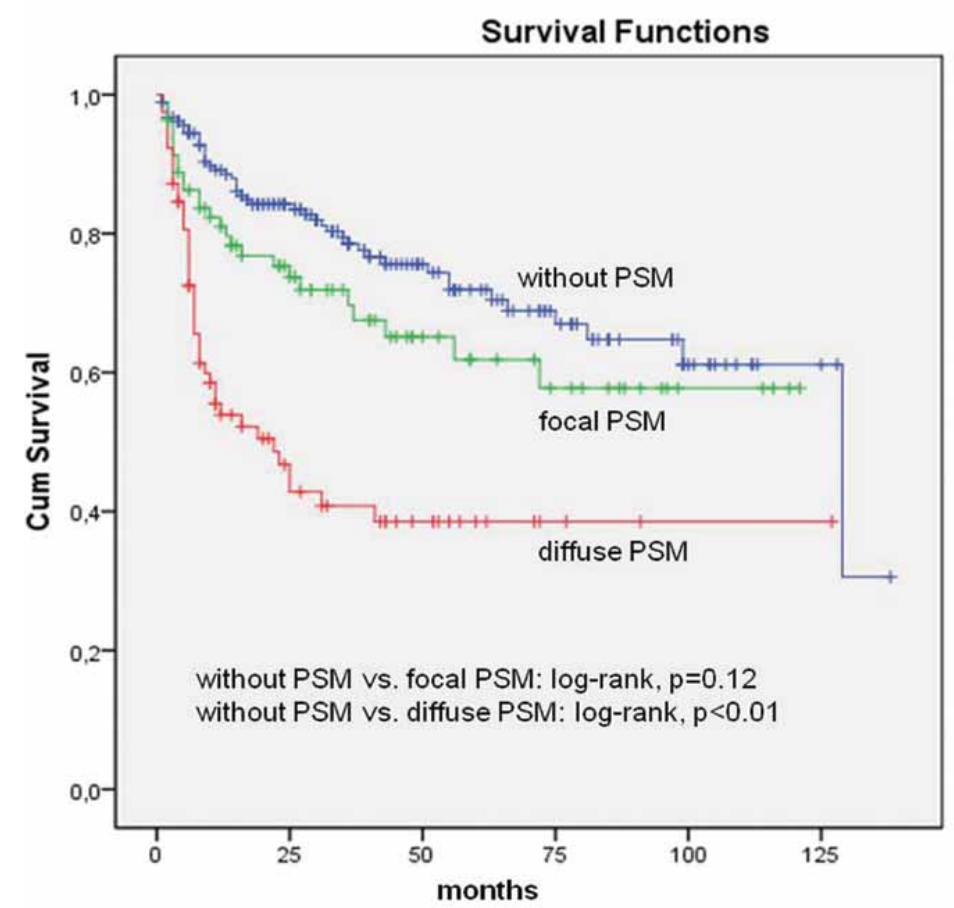


Table 3 - Cox proportional hazard analysis of several clinicopathological factors predicting time to biochemical recurrence following radical prostatectomy.

\begin{tabular}{lccc}
\hline Predictors & Hazard ratio $(95 \% \mathrm{Cl})$ & Wald test & P \\
\hline Univariate analysis & & & \\
Age & $0.99(0.97-1.02)$ & 0.22 & 0.64 \\
Focal EEP (Group 1) & $1.01(0.53-1.92)$ & $<0.01$ & 0.98 \\
Focal PSM (Group 1) & $1.47(0.91-2.38)$ & 2.41 & 0.12 \\
Diffuse EEP (Group 2) & $1.48(1.22-1.80)$ & 15.17 & $<0.01$ \\
Diffuse PSM (Group 2) & $3.45(2.25-5.30)$ & 32.18 & $<0.01$ \\
Preoperative PSA & $1.05(1.03-1.07)$ & 27.15 & $<0.01$ \\
Seminal vesicle invasion & $2.74(1.72-4.38)$ & 17.81 & $<0.01$ \\
RP Gleason score & $1.44(1.14-1.81)$ & 9.28 & $<0.01$ \\
RP tumor extent & $1.01(1.00-1.01)$ & 8.09 & $<0.01$ \\
Multivariate analysis & & & $<0.01$ \\
Diffuse PSM (Group 2) & $1.71(1.37-2.13)$ & 22.48 & $<0.01$ \\
Preoperative PSA & $1.04(1.02-1.06)$ & 11.64 & 0.04 \\
Seminal vesicle invasion & $1.79(1.02-3.14)$ & 4.10 & \\
\hline
\end{tabular}

study is in accordance with a more favorable biochemical progression following radical prostatectomy for patients with focal EPE than patients with extensive EPE $(1,4,6)$. In the 7 th edition of the TNM Classification of Malignant Tumours (15), the bladder neck involvement is considered pT3a and not pT4. Therefore, involvement of the bladder neck was considered EPE in this study. The incidence of EPE in the literature ranges from $28 \%$ to $48 \%$ (16). In our study the frequency was $35.8 \%$.

Epstein et al. (1) subdivided extraprostatic extension into focal and established. Focal extraprostatic extension was defined as a few glands immediately exterior to the prostate in 1 to 2 sections. Cancers with established EPE had a higher risk of progression than those with focal EPE. Wheeler et al. (4) defined focal EPE as tumor outside the prostate to a depth of less than one high-power field on no more than two separate sections and established EPE any amount of extraprostatic tumor more than focal. Extensive EPE was associated with progressively increased risk of seminal vesicle invasion and positive lymph nodes and lower progression free probabilities. Sung et al. (6) measured EPE considering the radial distance defined as the distance that the tumor protrudes perpendicular beyond the outer margin of the prostatic stroma. In univariate analysis, the radial distance of extraprostatic tumor measured by ocular micrometer was associated with PSA recurrence. In the multivariate analysis, radial distance remained an independent predictor of PSA recurrence.

Surgical margin status is a major predictor of biochemical recurrence following radical prostatectomy. Coupled with other variables might serve to further discriminate those patients 
most likely to benefit from immediate adjuvant radiotherapy after RP. Our results are in accordance with most studies that correlate extent of margin positivity in radical prostatectomies with biochemical recurrence (2,3,5,7-10,16-18). Diffuse PSM was significantly associated to shorter time of biochemical progression and was predictive of time to TBCR in both univariate and multivariate analyses. The incidence of PSM in the literature ranges from 16\% to $50 \%$ (16). In our study the incidence was $44.4 \%$. One of the largest potential sources of discrepancy for incidence of PSM is the different methods used to process the radical prostatectomy specimens (2).

Epstein et al. (2) defined focal margin positivity as margins in which the tumor reached the inked transected exterior surface, and the involved site(s) were limited and present in only one or two areas. Cases with extensive positive margins were those in which multiple positive margins were present at different sites in the prostate. Forty-one percent of the prostate specimens studied had positive margins, 37\% had focally positive margins, and $4 \%$ had extensive margins. The difference in biochemical progression in patients with focally positive versus extensively positive margins was significant. Weldon et al. (3) defined focal positive margins those present on a single step-section and $3 \mathrm{~mm}$ long or less. Any larger positive margins were designated as extensive. Forty-four percent of the specimens had positive surgical margins, 23\% focal and 21\% extensive. The difference in biochemical progression between patients with focal and extensive positive margins was significant. Babaian et al. (5) stratified PSM into focal and diffuse using a cutoff of $3 \mathrm{~mm}$ linear extent adding the length of all areas of tumor in contact with ink. The biochemical failure rate was 10\% when the positive margin length was $\leq 3 \mathrm{~mm}$ and $77.8 \%$ when the positive margin length was $>3 \mathrm{~mm}$. The authors concluded that the result stratify the probability of failure and suggested that adjuvant local therapy may be beneficial if the positive margin exceeds $3 \mathrm{~mm}$.

During a consensus conference held by the ISUP, no consensus could be reached as to how evaluate extent of EPE and PSM $(11,12)$. The conference recommended that until such a consensus is reached, EPE and PSM should be reported as millimeters of linear involvement. However, mea- surement is tedious, time consuming and not used in the daily practice of pathologists. Our proposal of EPE and PSM quantitation does not need any time consuming measurement. It implies only to consider the number of quadrants and/or sections from the bladder neck or apex showing EPE and/ or PSM and can be used in the daily practice of all pathologists who step-section and totally process the surgical specimen.

The present proposal of diffuse PSM was significantly predictive of TBCR following surgery. Only patients with diffuse PSM showed significantly shorter time to BCR. This difference was also significant considering only specimens with pathologic stage T2 as well as specimens pT2 and Gleason score $\leq 6$. No influence was found according to the location of PSM as well as weight of the prostate which influences the number of transversal sections. In the present cohort of patients, diffuse positive surgical margins were the strongest predictors of TBCR in univariate and multivariate analyses. It is interesting to point out why patients with positive margins may not inevitably progress to BCR. The main reason may be local tissue destruction as a result of the effects of disrupted vasculature, cautery, and inflammation related to surgery (17).

A limitation of the present study is the relatively short mean follow-up time. Johansson et al. (19) reported that local tumor progression and distant metastases developed even after 15 years of follow-up. However, it is recognized that over $90 \%$ of patients submitted to radical prostatectomy will have a relapse within 5 years after surgery $(19,20)$. Therefore, it is likely that the majority of cases with biochemical progression have already been identified in this study. It is also worth mention that Gleason grade in multivariate analysis was not a significant predictor of TBCR due to the small number of patients with score 8-10 $(\mathrm{n}=21)$. If Gleason grade was significantly predictive, then maybe the significance of some of the multivariate models tested would have been different.

\section{CONCLUSIONS}

In this study we showed a statistically significant predictive value for shorter time to 
biochemical progression following radical prostatectomy in patients with diffuse EPE or PSM using a simple method for EPE and PSM extent quantitation that can be used in the daily practice of all pathologists who step-section and totally process the surgical specimen. In univariate analysis but not in multivariate analysis, patients with EPE in more than 1 quadrant and/or section from the bladder neck or apex have a significant higher risk to develop biochemical recurrence in a shorter time. Patients with PSM in more than 2 quadrants and/or sections from the bladder neck or apex have a significant higher risk to develop biochemical recurrence in a shorter time in univariate and multivariate analyses. PSM was the strongest predictive finding for shorter time of recurrence in both analyses. We advocate reporting the extent of EPE and PSM as part of the surgical pathology report.

\section{ABBREVIATIONS:}

PSA: prostate specific antigen;

SD: standard deviation;

CI: confidence interval;

RP: radical prostatectomy;

BCR: biochemical recurrence;

TBCR: time to biochemical recurrence;

EPE: extraprostatic extension;

PSM: positive surgical margin;

ISUP: International Society of Urological Pathology;

USCAP: United States and Canadian Academy of Pathology.

\section{CONFLICT OF INTEREST}

None declared.

\section{REFERENCES}

1. Epstein JI, Carmichael MJ, Pizov G, Walsh PC: Influence of capsular penetration on progression following radical prostatectomy: a study of 196 cases with long-term followup. J Urol. 1993; 150: 135-41.

2. Epstein JI, Pizov G, Walsh PC: Correlation of pathologic findings with progression after radical retropubic prostatectomy. Cancer. 1993; 71: 3582-93.
3. Weldon VE, Tavel FR, Neuwirth H, Cohen R: Patterns of positive specimen margins and detectable prostate specific antigen after radical perineal prostatectomy. J Urol. 1995; 153: 1565-9. Erratum in: J Urol. 1995; 154: 538.

4. Wheeler TM, Dillioglugil 0, Kattan MW, Arakawa A, Soh S, Suyama K, et al.: Clinical and pathological significance of the level and extent of capsular invasion in clinical stage T1-2 prostate cancer. Hum Pathol. 1998; 29: 856-62.

5. Babaian RJ, Troncoso P, Bhadkamkar VA, Johnston DA: Analysis of clinicopathologic factors predicting outcome after radical prostatectomy. Cancer. 2001; 91: 1414-22.

6. Sung MT, Lin H, Koch MO, Davidson DD, Cheng L: Radial distance of extraprostatic extension measured by ocular micrometer is an independent predictor of prostate-specific antigen recurrence: A new proposal for the substaging of pT3a prostate cancer. Am J Surg Pathol. 2007; 31: 311-8.

7. Shikanov S, Song J, Royce C, Al-Ahmadie H, Zorn K, Steinberg $G$, et al.: Length of positive surgical margin after radical prostatectomy as a predictor of biochemical recurrence. J Urol. 2009; 182: 139-44.

8. van Oort IM, Bruins HM, Kiemeney LA, Knipscheer BC, Witjes JA, Hulsbergen-van de Kaa CA: The length of positive surgical margins correlates with biochemical recurrence after radical prostatectomy. Histopathology. 2010; 56: 464-71.

9. Resnick MJ, Canter DJ, Guzzo TJ, Magerfleisch L, Tomaszewski JE, Brucker BM, et al.: Defining pathological variables to predict biochemical failure in patients with positive surgical margins at radical prostatectomy: implications for adjuvant radiotherapy. BJU Int. 2010; 105: 1377-80.

10. Cao D, Humphrey PA, Gao F, Tao Y, Kibel AS: Ability of linear length of positive margin in radical prostatectomy specimens to predict biochemical recurrence. Urology. 2011; 77: 1409-14.

11. Magi-Galluzzi C, Evans AJ, Delahunt B, Epstein JI, Griffiths DF, van der Kwast $\mathrm{TH}$, et al.: International Society of Urological Pathology (ISUP) Consensus Conference on Handling and Staging of Radical Prostatectomy Specimens. Working group 3: extraprostatic extension, lymphovascular invasion and locally advanced disease. Mod Pathol. 2011; 24: 26-38.

12. Tan PH, Cheng L, Srigley JR, Griffiths D, Humphrey PA, van der Kwast $\mathrm{TH}$, et al.: International Society of Urological Pathology (ISUP) Consensus Conference on Handling and Staging of Radical Prostatectomy Specimens. Working group 5: surgical margins. Mod Pathol. 2011; 24: 48-57.

13. Cookson MS, Aus G, Burnett AL, Canby-Hagino ED, D'Amico AV, Dmochowski RR, et al.: Variation in the definition of biochemical recurrence in patients treated for localized prostate cancer: the American Urological Association Prostate Guidelines for Localized Prostate Cancer Update Panel report and recommendations for a standard in the reporting of surgical outcomes. J Urol. 2007; 177: 540-5. 
14. Billis A, Magna LA, Ferreira U: Correlation between tumor extent in radical prostatectomies and preoperative PSA, histological grade, surgical margins, and extraprostatic extension: application of a new practical method for tumor extent evaluation. Int Braz J Urol. 2003; 29: 113-9; discussion 120.

15. International Union Against Cancer (UICC). TNM Classification of malignant tumours, 7th ed, Sobin LH, Gospodarowicz M, Wittekind Ch (eds). Geneva:Wiley-Blackwell. 2009; pp. 243-8.

16. Watson RB, Civantos F, Soloway MS: Positive surgical margins with radical prostatectomy: detailed pathological analysis and prognosis. Urology. 1996; 48: 80-90.

17. Emerson RE, Koch MO, Jones TD, Daggy JK, Juliar BE, Cheng $L$ : The influence of extent of surgical margin positivity on prostate specific antigen recurrence. J Clin Pathol. 2005; 58: 1028-32.
18. Vis AN, Schröder FH, van der Kwast TH: The actual value of the surgical margin status as a predictor of disease progression in men with early prostate cancer. Eur Urol. 2006; 50: 258-65.

19. Johansson JE, Andrén 0 , Andersson SO, Dickman PW, Holmberg L, Magnuson A, et al.: Natural history of early, localized prostate cancer. JAMA. 2004; 291: 2713-9.

20. Kattan MW, Eastham JA, Stapleton AM, Wheeler TM, Scardino PT: A preoperative nomogram for disease recurrence following radical prostatectomy for prostate cancer. J Natl Cancer Inst. 1998; 90: 766-71.

Correspondence address: Dr. Athanase Billis Department of Pathology School of Medical Sciences - UNICAMP Rua Tessália Vieira de Camargo, 126 Campinas, SP, 13083-887, Brazil FAX: + 5519 3289-3897 E-mail: athanase@fcm.unicamp.br 